\title{
Analisi psicologica in un Centro di Dialisi
}

\author{
M. Colagreco, S. Mennilli, P. Cappelli, L. Di Liberato, M. Bonomini \\ Istituto di Clinica Nefrologica-Terapia Emodepurativa, Ospedale Clinicizzato SS. Annunziata, Chieti
}

\section{Introduzione}

La terapia dialitica (peritoneale ed extracorporea) è un peculiare metodo di cura che rappresenta, sul piano individuale, l'interruzione di un normale corso di vita e, soprattutto, una lacerazione della propria persona. Essa può creare importanti ripercussioni emotive sul paziente e su chi lo circonda quali ansia, depressione, paura, timori e sensi di colpa.

Per trattare in maniera adeguata i malati dializzati occorre occuparsi di questi loro numerosi problemi, ma anche delle difficoltà dei familiari e del possibile disagio degli operatori. Se tali problematiche psicologiche non vengono affrontate e risolte, possono alimentare un disagio psicologico continuativo che si esprime: a livello del paziente, con sintomi tipici quali prurito, dolore e insonnia, con comportamenti di passività e di rifiuto, con scarsa compliance al trattamento e con la comparsa di stati depressivi, ansiosi, sensazioni di abbandono ecc.; nel rapporto operatorepaziente, con sentimenti giudicanti, di inadeguatezza, di forti coinvolgimenti emotivi o forti distacchi e rifiuti da parte del paziente, con comportamenti neutrali verso tutti i malati, con cali dell'efficienza ecc.; nel rapporto tra gli operatori stessi con contrasti circa le competenze, formazione di sottogruppi, rigidità nei cambi di turni e orari, assenteismo ecc.

Lattività psicologica con i pazienti in trattamento dialitico ha come obiettivo il recupero di quelle dimensioni personali dell'individuo che costituiscono una risorsa nel supportare e facilitare il paziente ad affrontare la malattia e il trattamento a essa associato, integrando e potenziando l'intervento specificatamente medico.

Da qui l'importanza della figura dello psicologo all'interno di un reparto quale quello di Nefrologia e Dialisi, dove nell'ambito della psicologia della salute dovrebbero essere trattate tutte quelle manifestazioni di disagio psicologico che accompagnano ogni esperienza di malattia, così come la sofferenza emotiva e gli effetti della stessa sia nel paziente e nella famiglia che nel suo contesto di vita.

\section{Scopo dello studio}

È quasi inevitabile che, prima o dopo l'inizio del trattamento dialitico regolare, il paziente incontri una fase depressiva, ed è altrettanto inevitabile come chiunque lavori in contesti a elevata implicazione emotiva sia maggiormente soggetto a sviluppare burnout. Il burnout non è altro che un meccanismo di difesa che l'operatore mette in atto per far fronte al coinvolgimento sempre più pressante e profondo degli utenti; sono tali dinamiche psicologiche presenti nella relazione di aiuto ad avere la parte più importante nello sviluppo di tale sindrome, portando a bruciare le risorse psicologiche dell'individuo dal continuo e cronico contatto con emozioni forti.

Lo scopo del presente lavoro è quello di voler analizzare sia il livello di burnout negli operatori sanitari del Centro di Nefrologia e Terapia Emodepurativa dell'Ospedale Clinicizzato "SS. Annunziata" di Chieti, sia i livelli di ansia e depressione in alcuni dei pazienti sottoposti a trattamento emodialitico presso il Centro.

\section{Pazienti e Metodi}

\section{1) Valutazione Psicologica del burnout negli Operatori Sanitari}

Tutti i 17 operatori contattati ( 8 maschi, 9 femmine, di età compresa tra 25 e 54 anni, media 42 anni) hanno aderito, chi più chi meno, volentieri alla ricerca, il cui obiettivo era quello di analizzare il livello di stress e, pertanto, di burnout mediante somministrazione del test Maslach Burnout Inventory (MBI).

L'MBI è un test di autosomministrazione che necessita di 
circa 10-15 minuti per la sua compilazione. ̇̀ formato da 22 items, espressi in forma affermativa, circa atteggiamenti e sentimenti personali, divisi in tre sottoscale: ESAURIMENTO EMOTIVO, DEPERSONALIZZAZIONE, REALIZZAZIONE PERSONALE. L'operatore è tenuto a rispondere a ogni item attraverso una scala di valori da $0 \mathrm{a}$ 6 , in relazione all'intensità del sentimento provato verso quella specifica domanda:

\begin{tabular}{lcccccc}
\hline 0 & 1 & 2 & 3 & 4 & 5 & 6 \\
\hline $\begin{array}{c}\text { mai } \\
\text { qualche } \\
\text { volta } \\
\text { all'anno }\end{array}$ & $\begin{array}{c}\text { una volta } \\
\text { al mese }\end{array}$ & $\begin{array}{c}\text { qualche } \\
\text { volta } \\
\text { al mese }\end{array}$ & $\begin{array}{c}\text { una volta } \\
\text { alla } \\
\text { settimana }\end{array}$ & $\begin{array}{c}\text { qualche } \\
\text { volta } \\
\text { alla } \\
\text { settimana }\end{array}$ & $\begin{array}{c}\text { ogni } \\
\text { giorno }\end{array}$ \\
& & & & & & \\
\hline
\end{tabular}

Gli items che fanno riferimento alle tre sottoscale sono distribuiti in ordine sparso all'interno del test, al fine di non influenzare in alcun modo le risposte.

Gli items del MBI, suddivisi per ciascuna sottoscala, sono riportati nella Tabella I. In base ai punteggi ottenuti, si considera:
- un alto grado di burnout se sono presenti alti punteggi nelle sottoscale EE e Dp e bassi punteggi nella sottoscala Rp;

- un medio grado di burnout se sono presenti punteggi medi in tutte le sottoscale;

- un basso livello di burnout se sono presenti bassi punteggi nelle sottoscale EE e Dp e alti punteggi nella sottoscala Rp.

È importante ricordare che l'MBI è un test anonimo, e ciò è stato sempre specificato prima della consegna di ogni copia a ciascun operatore. Inoltre, per far sì che il test fosse il più possibile aderente alla realtà, la somministrazione è avvenuta secondo alcuni criteri, quali la riservatezza e la discrezione, oltre all'anonimato.

\section{2) Valutazione psicologica di ansia e depressione nei pazienti in trattamento emodialitico}

Il test psicologico viene definito come una misura obiettiva e standardizzata di un campione di comportamento.

Nel presente studio sono state sottoposte due batterie di test a pazienti in emodialisi regolare: l'ASQ, atto a valutare i livelli di ansia (sia manifesta che latente) presenti nel soggetto, e il $\mathrm{CDQ}$, quale misuratore degli stati depressivi.

TABELLA I - ITEMS DEL MASLACH BURNOUT INVENTORY (MBI) TEST

SOTTOSCALA

ESAURIMENTO EMOTIVO (EE)

DEPERSONALIZZAZIONE (Dp)

REALIZZAZIONE PERSONALE (Rp)

\section{ITEM}

1. Mi sento emotivamente sfinito dal mio lavoro

2. Mi sento sfinito alla fine di una giornata di lavoro

3. Mi sento stanco quando mi alzo la mattina e devo affrontare un'altra giornata di lavoro

6. Mi pare che lavorare tutto il giorno con la gente mi pesi

8. * Mi sento esaurito dal mio lavoro

13. Sono frustrato dal mio lavoro

14. Credo di lavorare troppo duramente

16. Lavorare direttamente a contatto con la gente mi crea troppa tensione

20. Sento di con farcela più

5. Mi pare di trattare alcuni utenti come se fossero degli oggetti

10. ${ }^{\star}$ Da quando ho cominciato a lavorare qui sono diventato più sensibile con la gente

11. *Ho paura che questo lavoro mi possa indurire emotivamente

15. *Non mi importa veramente di ciò che succede ad alcuni utenti

22. Ho l'impressione che alcuni utenti diano la colpa a me per i loro problemi
4. Posso capire facilmente come la pensano i miei utenti
7. *Affronto efficacemente i problemi dei miei utenti
9. Credo di influenzare positivamente la vita di altre persone attraverso il mio lavoro
12. Mi sento pieno di energie
17. * Riesco facilmente a rendere i miei utenti rilassati e a proprio agio
18. Mi sento rallegrato dopo aver lavorato con i miei utenti
19. Ho realizzato molte cose di valore nel mio lavoro
21. Nel mio lavoro affronto i problemi emotivi con calma

*Item di maggiore significatività nelle sottoscale di riferimento 
Sono stati esaminati 11 pazienti ( 6 maschi, 5 femmine) di età compresa tra 42 e 78 anni (media 61.5 anni). Tra i tanti pazienti in cura presso il reparto, diversi sono stati quelli che hanno manifestato delle resistenze e quelli che, invece, senza sapere neppure cosa si volesse analizzare attraverso l'elaborazione dello stesso, hanno rifiutato a priori. La scelta, comunque, è risultata libera e priva di ogni tipo di vincolo.

L'ASQ è stato costruito per dare uno strumento che permetta di ottenere, in maniera rapida, oggettiva e standardizzata, una informazione clinica dell'ansietà. E un questionario costituito da 40 items, che danno una stima del livello di ansietà, e sono un prezioso aiuto alla diagnosi clinica. La misurazione può essere usata non solo per una diagnosi iniziale, ma anche nel follow-up, come successivo controllo, per verificare se sono intervenuti progressi in seguito al trattamento psicoterapeutico, o al cambiamento di situazioni ecc.

L'elaborazione del questionario è avvenuta attraverso l'autosomministrazione (in un tempo di circa 15') perché, diversamente, si sarebbero potute condizionare le risposte date dai pazienti, in quanto qualsiasi esaminato tende a dare un'immagine diversa, tendenzialmente migliore, da quella che è agli occhi dell'esaminatore.

Il CDQ è lo strumento che, con facilità, permette l'individuazione della quantità e del tipo di depressione in corso nel soggetto, dove per depressione si intende un insieme di sintomi che consistono nell'abbassamento del tono dell'umore, tristezza, rammarico, nostalgia, generico pessimismo, inibizione motoria e psichica ecc.

La decisione di portare attenzione particolare alle situazioni depressive è data da due ragioni principali:

1. normalmente l'inizio del trattamento dialitico induce depressione e, se il soggetto è già tendenzialmente depresso, la situazione può degenerare in vere e proprie forme nevrotiche;

2. perché, ma non è riferito a questo studio, essa rappresenta uno dei parametri che vengono più comunemente utilizzati per la scelta del tipo di dialisi più idonea al paziente, dove si prende in considerazione la tendenza del soggetto a sviluppare, o meno, stati depressivi.

Tale test, somministrabile in circa 15', è composto da 40 items; il suo utilizzo, correlato a quello dell'ASQ e all'MMPI, è particolarmente utile nella fase dei primissimi colloqui col paziente quando, non ancora sottoposto a nessun tipo di trattamento dialitico, viene scelto quello più consono a lui in base ai risultati dei suddetti test, al parere medico e, soprattutto, alle volontà che il paziente stesso manifesta.

Anche per il CDQ l'elaborazione è avvenuta attraverso l'autosomministrazione.

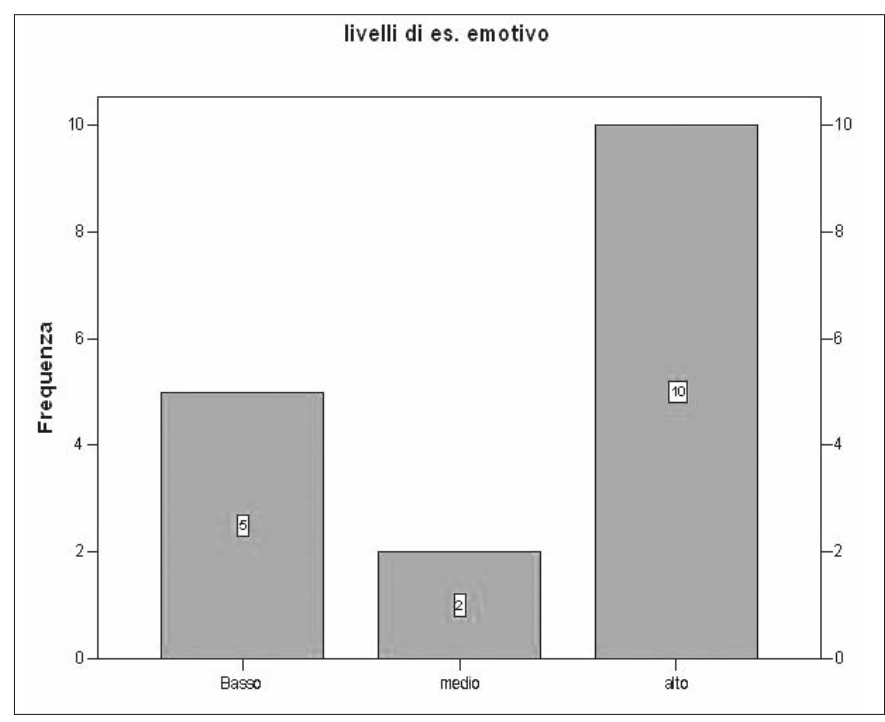

Fig. 1 - Livelli di esaurimento emotivo negli operatori sanitari sottoposti al Maslach Burnout Inventory test.

\section{Risultati}

\section{1) Analisi psicologica negli operatori sanitari}

Gli operatori hanno reagito con sentimenti differenti alla consegna del test; alcuni entusiasti, altri disponibili e, infine, la minoranza, come se obbligati.

Tra gli entusiasti si riscontrano gli operatori più giovani anagraficamente, con un maggior impeto nello svolgere le diverse mansioni a contatto con l'utenza.

Gli operatori disponibili hanno fatto molte domande circa la ricerca, gli scopi e la curiosità nel conoscere anche la disponibilità degli altri colleghi, mentre l'ultima categoria sembrava quasi compilare il test in maniera distaccata, perplessi e diffidenti dall'uso del questionario come rivelatore di indici importanti per il reparto e per loro stessi.

Il test MBI somministrato agli operatori sanitari si compone di 3 sottoscale: esaurimento emotivo, depersonalizzazione e realizzazione personale (Tab. I).

Per Esaurimento Emotivo si intende la particolare sensazione di inaridimento emotivo e logoramento. Una persona si sente coinvolta in modo eccessivo dal punto di vista delle emozioni e viene sopraffatta dalle richieste emozionali che gli altri gli impongono.

Dall'analisi dei dati, emerge che il 50\% dei soggetti del campione in esame presenta un livello alto di tale esaurimento (Fig. 1), dato alquanto significativo se sommato al livello medio rappresentato dal 10\% dei soggetti. Questo dato è indice di un livello elevato di stress lavorativo del campione che deve essere valutato alla luce degli altri indici di seguito riportati. 


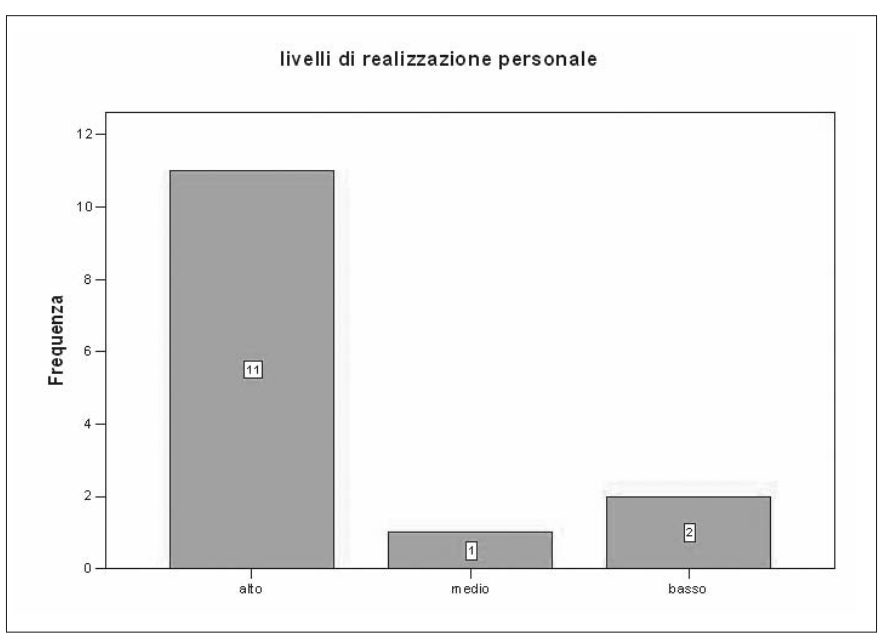

Fig. 2 - Livelli di realizzazione personale negli operatori sanitari sottoposti al Maslach Burnout Inventory test.

La Realizzazione Personale è la sensazione di competenza che avverte il soggetto e il desiderio di successo nel lavorare con gli altri. Bassi livelli di questo fattore danno adito alla sensazione che nel lavoro a contatto con gli altri la propria competenza e il proprio desiderio di successo stiano venendo meno. Il campione in esame presenta una percentuale di realizzazione personale pari al 55\% (Fig. 2), e ciò rappresenta un fattore positivo nella prevenzione del rischio burnout su cui far leva in eventuali corsi e trattamenti di supporto. Tuttavia va considerato che le omissioni da parte di alcuni soggetti ad alcune domande del questionario potrebbero inficiare, anche se parzialmente, l'attendibilità dei dati.

La Depersonalizzazione valuta il comportamento dell'operatore nei confronti dell'utente. $\grave{\mathrm{E}}$, in pratica, la risposta negativa nei confronti delle persone che ricevono la prestazione professionale. Nel campione in esame (Fig. 3), i soggetti che presentano una tale problematica sono solo 4 su 16 (e non 17 per via delle omissioni già citate) e rappresentano, quindi, una percentuale minima. Tale fattore però, rappresenta un importante indice di rischio perché racchiude in sé proprio la sintomatologia tipica del burnout, e pertanto va analizzato scrupolosamente (si deve, però, considerare che tale fattore può anche rappresentare proprio una caratteristica di personalità o di comportamento tipica del soggetto esaminato, e pertanto non riconducibile alla sintomatologia tipica del burnout).

I dati ottenuti nel test MBI sono successivamente stati analizzati attraverso indagini statistiche specifiche (Tavole di contingenza, test chi-quadrato di Pearson) atte a valutare la possibilità di rischio della sindrome del burnout in rapporto ad alcune variabili. In particolare, è stato valutato se esiste una dipendenza significativa tra livelli di esaurimento emotivo, depersonalizzazione e realizzazione

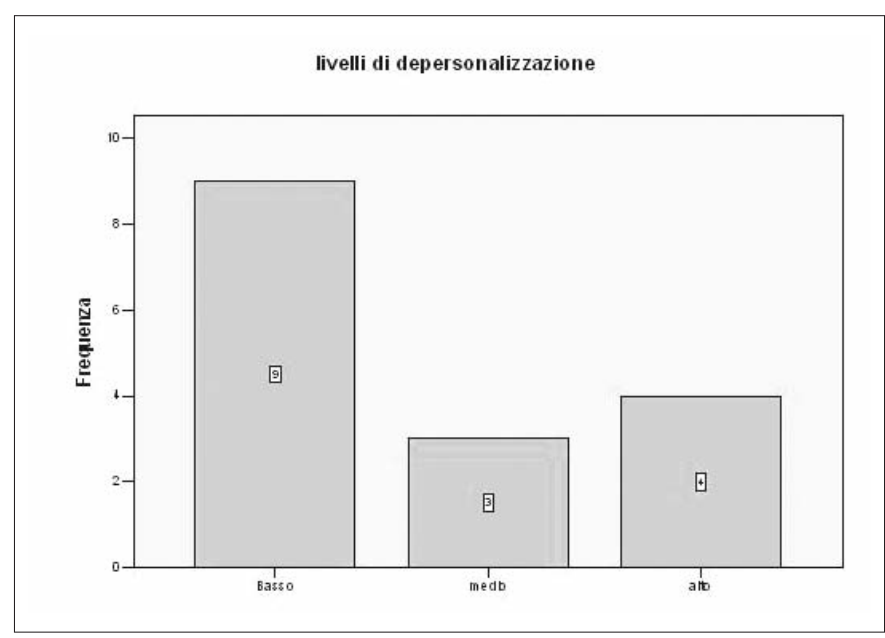

Fig. 3 - Livelli di depersonalizzazione negli operatori sanitari sottoposti al Maslach Burnout Inventory test.

personale con le ore di lavoro settimanale, il tempo di attività lavorativa complessiva e il tempo di attività lavorativa nel reparto di Nefrologia e Dialisi.

E stata riscontrata una correlazione statisticamente significativa, quindi una stretta dipendenza, tra ore di lavoro settimanali e livelli elevati di esaurimento emotivo $(p=0.007)$ e depersonalizzazione $(p=0.009)$. Al contrario $i$ livelli di realizzazione personale non sono risultati correlarsi con le variabili valutate, a indicare che probabilmente la motivazione al lavoro è piuttosto alta.

\section{2) Analisi psicologica nei pazienti emodializzati}

A 11 pazienti in trattamento emodialitico periodico sono stati somministrati il test ASQ (che valuta i livelli di ansia) e il test CDQ (misuratore degli stati depressivi).

Analizzando i risultati ottenuti nel test ASQ, è stato realizzato un grafico (Fig. 4) dove è stato possibile analizzare l'andamento delle risposte date da ciascun soggetto. I due indici posti sull'ascissa e sull'ordinata rappresentano rispettivamente l'indice dell'età e il valore dello sten (i 10 valori stens rappresentano categorie standardizzate di punteggi ottenuti mediante una trasformazione lineare dei punti grezzi, utili per la comparazione dei risultati). Occorre considerare che:

punteggi sten di 1, 2, 3 si trovano in soggetti eccessivamente rilassati, calmi, flemmatici;

generalmente, un punteggio sten di 4, 5, 6 o 7 indica un livello medio di ansietà;

un punteggio sten di 8 indica una persona cui il livello di ansietà può diventare serio;

punteggi sten di 9 e 10 si trovano solo in una persona ogni venti.

L'andamento delle risposte date da ciascun soggetto al test 


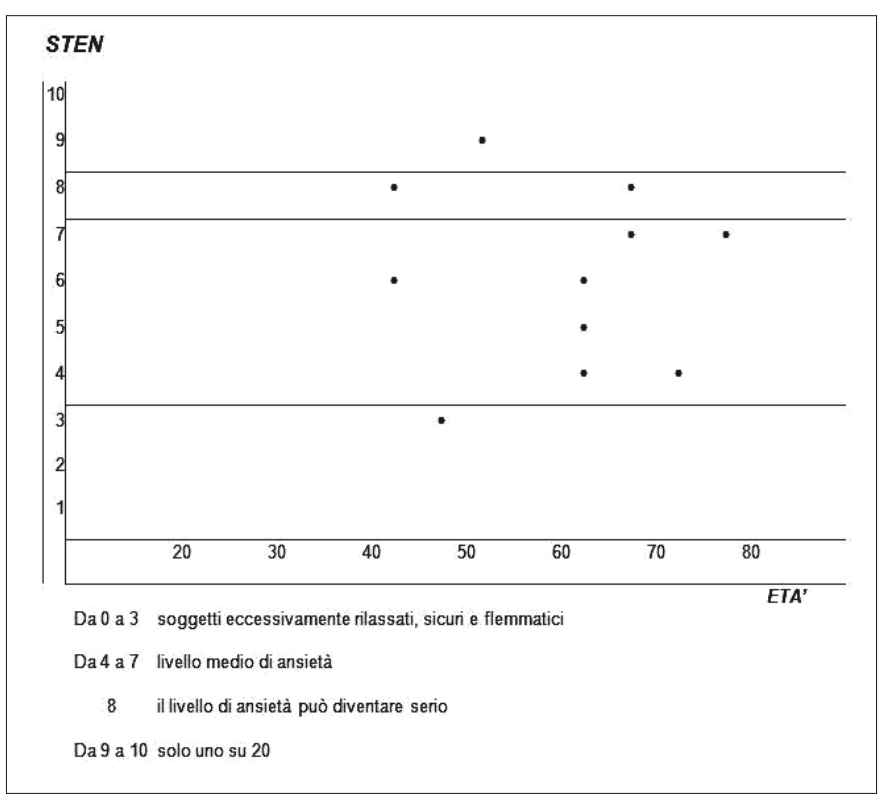

Fig. 4 - Test ASQ nei pazienti emodializzati.

$\mathrm{CDQ}$ è riportato nella Figura 5. I due indici posti sull'ascissa e sull'ordinata rappresentano rispettivamente l'indice dell'età e il valore dello sten. Per ottenere i punti stens, sono state utilizzate tavole di conversione particolari, elaborate secondo gruppi specifici, in quanto se si fossero utilizzate tabelle di conversione classiche (definite "regolari") si sarebbero ottenuti pochi punteggi stens bassi (cioè dall'1 al 5) e un numero molto grande di punteggi alti (dal 6 al 10) e questo non per un difetto delle tavole, ma semplicemente per il fatto che i soggetti clinici, nel complesso, raggiungono una deviazione standard superiore ai soggetti normali. Ecco che la scelta della tavola di norma diventa fondamentale perché può avere un valore particolare nell'interpretazione del punteggio del test.

Punteggi sten di 1, 2, 3 si trovano in soggetti eccessivamente rilassati, piatti, flemmatici.

Anche per questo test, un punteggio sten di 4, 5, 6 o 7 indica un livello medio di depressione.

Un punteggio sten di 8 indica una persona cui il livello di depressione può diventare serio.

Punteggi sten di 9 e 10 si trovano solo su una persona ogni venti.

\section{Discussione}

\section{Considerazioni sul burnout negli operatori del Centro Dialisi}

Questo studio, se pur limitato, ha permesso di racchiudere tutte le difficoltà che gli operatori socio-sanitari di un

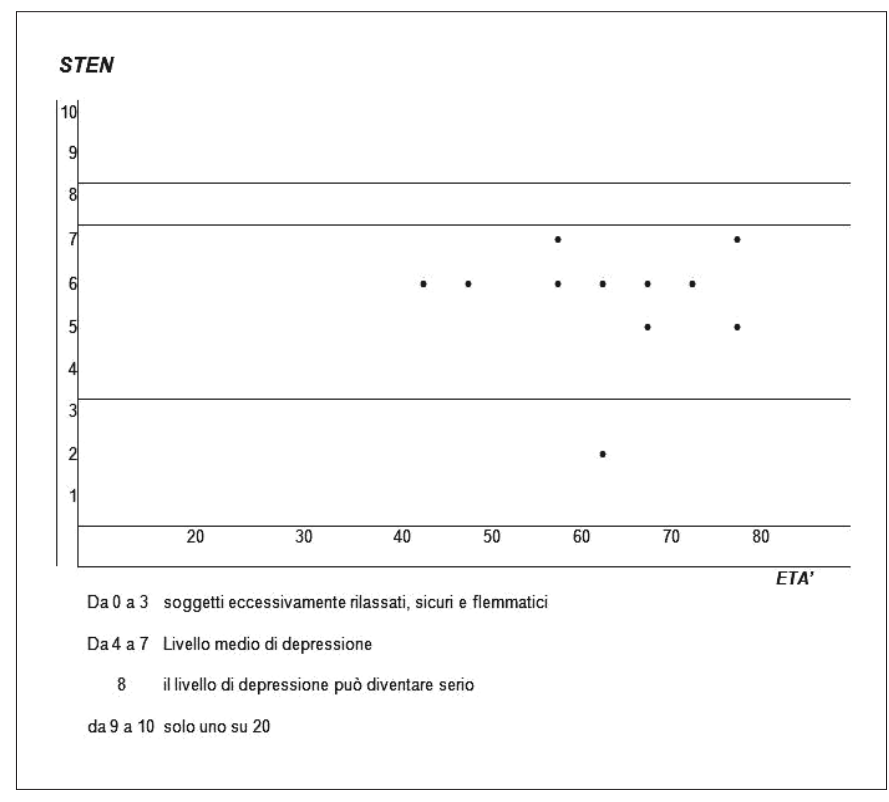

Fig. 5 - Test CDQ nei pazienti emodializzati.

Centro Dialisi possono incontrare nello svolgimento del loro lavoro quotidiano.

Tra i soggetti esaminati, quelli ad alto rischio sono risultati 2 e a medio 4, il 35\% circa dei lavoratori. Ciò va opportunamente esaminato caso per caso, cercando di approfondire meglio e comprendere quali siano le problematiche in atto e i provvedimenti da adottare prima che la situazione peggiori ulteriormente.

Le direttive per risolvere o prevenire il burnout devono focalizzarsi sull'individuo, sul suo contesto lavorativo e sulle relazioni interpersonali. Viene richiesta molta importanza nella cura e salute del corpo e dello spirito, migliorando la conoscenza di sé; molto spesso, però, ciò non basta e, per tali ragioni, bisogna ricorrere a modificare l'ambiente o il proprio stile di lavoro.

Il burnout è fortemente correlato allo stress, e pertanto è importante ridurre quest'ultimo con corsi di formazione che rendano competenti e capaci di gestire le nuove situazioni, le relazioni e il lavoro insieme. Bisogna anche imparare a dialogare, in quanto è proprio alla base delle relazioni interpersonali (tra colleghi, malati, superiori) che si rintracciano le principali fonti di burnout.

Una intuizione che ha dato buoni risultati nella prevenzione e cura del burnout è stata la creazione e gestione, da parte di personale specializzato, di luoghi e punti di ritrovo dove gli operatori potessero riversare le proprie ansie, sofferenze e interrogativi ad altri impegnati nello stesso loro compito, e quindi capaci di offrire loro un valido aiuto. Qui c'è la possibilità anche di lavorare sui propri ideali, sulle aspettative e sulle domande che gli altri hanno o che loro stessi portano sul loro lavoro. 
La sofferenza è data proprio dallinfrangersi, per ciascun operatore impegnato nella professione di aiuto, di quel compito di "guaritore onnipotente" che ognuno crede di possedere. Ed ecco che per questo bisogna considerare lideale del guarire/combattere/risolvere con quello più consono e comprensibile per il malato del prendersi cura/sollevare/aiutare, unendolo al saper fare di ciascuno, non solo tecnico ma anche relazionale.

Dare significati nuovi al proprio lavoro significa non solo modificare il rapporto col malato e influire sul suo benessere globale ma anche, e soprattutto, agire sui propri vissuti, sul proprio benessere psicologico e, pertanto, prevenire una buona dose di stress e di burnout.

\section{Considerazioni sui test ASQ e CDQ nei pazienti in trat- tamento emodialitico}

Il modo più semplice per capire il significato di un punteggio dell'ASQ, e quindi conoscere meglio le problematiche che presenta il soggetto esaminato, è di analizzare il contenuto di ciascun item del test e così vedere come la persona più ansiosa descrive il proprio comportamento e come percepisce il comportamento altrui.

Analizzando ciascun item, si è potuto osservare come i tre pazienti che hanno ottenuto punteggi più alti al test siano concordi in alcune risposte date nel questionario. Le domande che hanno ottenuto risultati affermativi per tali soggetti sono state le seguenti:

12. da bambino avevo paura del buio;

15. mi disturba quando mi vengono mosse delle critiche anche se fatte per aiutarmi;

28. molte persone sono un po' strane, ma non lo vogliono ammettere;

38. mi viene da tremare e da sudare quando penso di dover affrontare un compito difficile;

40. qualche volta sono teso e confuso se penso alle cose che mi coinvolgono.

Altre risposte a cui i pazienti hanno dato punteggi affermativi, anche se alcuni di loro non sempre avevano votazioni massime, sono state le seguenti:

2. anche se la gente ha poca stima di me, io continuo ad essere fiducioso in me stesso;

5. se potessi incominciare di nuovo la mia vita, l'organizzerei in modo del tutto diverso;

8. dubito della sincerità della gente che si dimostri più cordiale di quanto mi aspetti;

13. a volte mi dicono che quando sono agitato, lo dimostro troppo apertamente nel modo di parlare o nel modo di comportarmi;

16. spesso mi arrabbio troppo facilmente con gli altri;

18. qualche volta dubito che le persone alle quali parlo siano veramente interessate a quello che dico;
20. nel discutere con certe persone, sono così agitato da non fidarmi più della verità di ciò che dico;

21. quando devo fare qualche cosa, impiego più energie della maggior parte delle persone, perché divento teso e nervoso;

30. mi sento di cattivo umore e non desidero vedere gente; 33. mi sveglio di notte e per le preoccupazioni faccio fatica a riprendere sonno.

Come si può osservare dai risultati ottenuti, il soggetto ansioso riporta un grado di irrequietezza, reattività fobica $\mathrm{e}$ sospettosità più alti del normale. Esso non si cura dei particolari, quantunque solo apparentemente, cioè trattandosi del proprio benessere fisico, poiché più sensibile, o per lo meno più insoddisfatto, della vita di un individuo meno ansioso e meglio adattato.

Le caratteristiche principali su cui esaminato ed esaminatore concordano sono: tensione / instabilità emotiva/ apprensione / mancanza di controllo / sospettosità, quali cinque tratti che compongono l'ansia nella sua veste più generale, nonché i cinque parametri specifici che il test prende in considerazione.

I soggetti esaminati che hanno manifestato punteggi più alti, a un'analisi più dettagliata hanno rilevato caratteristiche quali l'apprensione, l'autoaccusarsi, l'insicurezza, l'agitazione e la preoccupazione, quali fattori di ansia manifesta. Sono tesi, frustrati, agitati ed esausti, ma tali aspetti si evidenziano a un'analisi dell'ansia latente, quindi con caratteristiche non direttamente osservabili nel soggetto, che non appare incontrollato o preso da atteggiamenti impulsivi e presenta emozioni che appaiono relativamente stabili e realistiche. La loro sospettosità, gelosia e diffidenza verso l'altro è molto forte, anche se prevale maggiormente a livello latente.

Per i tre pazienti che hanno ottenuto punteggi più alti al test, si è deciso di valutare la veridicità delle risposte attraverso un colloquio a due, tra paziente e psicologo, al fine di controllare i vari items che hanno alzato il punteggio e di analizzare direttamente lo stato ansioso del paziente, per poter trovare delle soluzioni a riguardo e aiutarlo il più possibile a riportare il livello d'ansia a parametri di normalità.

I tre colloqui sono stati molto importanti; si è potuto valutare quanto il test fosse stato valido e attendibile nell'analizzare i livelli di ansia e soprattutto quali fossero le richieste dei pazienti in riferimento a questa problematica che, in un periodo di sei mesi, solo uno di loro aveva manifestato direttamente durante i colloqui in reparto (c'è da dire che la maggior parte di loro difficilmente parla di problemi personali durante il trattamento per mantenere una certa segretezza verso gli altri pazienti con i quali condivide la stanza di terapia).

Per ciò che concerne il punteggio risultante dal test per 
la depressione, il CDQ, a una prima analisi è possibile osservare come i valori depressivi per ciascun paziente siano tutti contenuti nella fascia compresa tra 4 e 7 , indicante un livello medio di depressione. Ciò sta a indicare come, in questi 11 pazienti sottoposti a valutazione, nessuno abbia livelli depressivi preoccupanti o tali da consigliarci un intervento da realizzare a breve termine.

Pertanto i colloqui individuali non sono stati fatti e a ciascun paziente è stato comunicato, così come per il test precedente, l'esito di ciascuna valutazione.

I test utilizzati sono stati considerati solo come ausilio e strumento conoscitivo; questi non possono e non devono essere disgiunti da colloqui e da una corretta ricerca anamnestica al fine di poter supportare ed elaborare, sia a livello cognitivo che emotivo, l'esperienza di malattia di ciascun paziente.

\section{Conclusioni}

La figura professionale dello psicologo all'interno di un reparto come quello di Nefrologia e Dialisi, a stretta valenza emotiva, è quasi assente in numerose realtà ospedaliere.
Incentivare il contributo psicologico porterebbe alla costruzione di una proficua collaborazione professionale, atta alla realizzazione di numerosi interventi mirati soprattutto al raggiungimento di due obiettivi: il valorizzare i bisogni individuali e le differenze che caratterizzano il vissuto di malattia di ciascun paziente, aiutandoli pian piano a ritrovare loro stessi e la loro vita, e il facilitare il personale sanitario nel riconoscere e regolare, nella relazione interpersonale, tali aspetti in modo più funzionale rispetto agli obiettivi terapeutici, in base anche alla consapevolezza circa il proprio vissuto emotivo.

colagrecom@libero.it

\section{Bibliografia di riferimento}

- Tummino V. Dialisi e bisogni psicologici: modello integrato di intervento sul paziente con insufficienza renale cronica di grado severo e i suoi familiari. Da: Progetto HPH - Macro Area: ACCOGLIENZA, Regione Lombardia - Azienda Ospedaliera Sant'Anna Como. Disponibile su: http://www.hsacomo.org/v2/ download $/$ ?file $=6$

- Trabucco G. Emodialisi e bisogni psicologici - Aspetti teorici, problemi psicologico-clinici. Quaderni di Psicoin: Modelli di intervento dello psicologo in ospedale, confronto di esperienze. A cura di Roberto Ferretti e Maria Virginia Gentili. Disponibile su: http://www. sipsot.it/html/ricercafolder/ricerca_ospedaliera/ documenti/emodialisi/Emodialisi.html

- Trabucco G. I problemi psicologici degli emodializzati. Atti XI Corso Nazionale di Aggiornamento Tecnici Emodialisi, Seminario Ante 2003. Disponibile su: http://
www.ante.it/atti/Corso2003/Trabucco2003.html

- Fuiano G, Caglioti A, Marino F, et al. L’insufficienza renale acuta nell'anziano. G Ital Nefrol 2001; 18: 469-81. Disponibile su: http://www.sin-italy.org/educational/ sinphony/docs/testi/nefro/conferenze/fuiano_GIN_2001_ 18_469-481_conf.pdf

- AAVV. Verso l'insufficienza. Dialisi peritoneale e qualità di vita. CENSIS, 1998.

- Maslach C. M.B.I., Maslach Burnout Inventory. Adattamento italiano: Pedrabissi L. e Santinello M., Firenze: O.S. Organizzazioni Speciali, 1999.

- Krug SE, Scheier IH, Cattel RB. Questionario di autovalutazione ASQ (Ipat Anxiety Scale). Adattamento italiano: Novaga M. e Pedon A., Firenze: O.S. Organizzazioni Speciali, 1979.

- Krug SE, Laughlin JE. Questionario di autovalutazione CDQ (Ipat Depression Scale). Adattamento italiano Novaga M. e Pedon A., Firenze: O.S. Organizzazioni Speciali, 1978. 